\title{
Effect of Weak Magnetic Intergranular Phase on the Coercivity in the HDDR Nd-Fe-B Magnet
}

\author{
M. LIU, G. B. HAN, R. W. GAO \\ School of Physics, Shandong University, Jinan, China. \\ Email: hangb@sdu.edu.cn
}

Received July $2^{\text {nd }}, 2009$; revised August 11 ${ }^{\text {th }}, 2009$; accepted August $19^{\text {th }}, 2009$.

\begin{abstract}
Assuming that intergranular phase (IP) existing between adjacent grains is a weak magnetic phase, we study the effect of IP on the coercivity in the HDDR Nd-Fe-B magnet. The results indicate that the coercivity increases with the increasing IP's thickness $d$, but decreases with increasing its anisotropy constant $K_{1}(0)$. When the structure defect thickness $r_{0}=6 \mathrm{~nm}, d=1 \mathrm{~nm}$ and $K_{1}(0)=0.15 K_{1}\left(K_{1}\right.$ is the normal anisotropy constant in the inner part of a grain), our calculated coercivity is in agreement with available experimental data.
\end{abstract}

Keywords: HDDR Nd-Fe-B Magnet, Intergranular Phase, Coercivity

\section{Introduction}

The HDDR powder particles, prepared by the HDDR (hydrogenation, decomposition, desorption, and recombination) process, consist of fine $\mathrm{Nd}_{2} \mathrm{Fe}_{14} \mathrm{~B}$ crystalline grains with diameters ranging from 0.2 to $0.3 \mu \mathrm{m}$, which is close to the single domain size of $\mathrm{Nd}_{2} \mathrm{Fe}_{14} \mathrm{~B}$ phase [1]. Such unique grain microstructure of HDDR magnet is different from not only the grain microstructure of sintered magnet, but also that of nanocrystalline magnet. Generally, the sintered magnet consists of the $\mathrm{Nd}_{2} \mathrm{Fe}_{14} \mathrm{~B}$ crystalline grains of 5-10 $\mu \mathrm{m}$ in diameter, and nonmagnetic Nd-rich boundary phases [2] which interrupts the intergrain exchange coupling interaction. Thus, the grainboundary anisotropy (GBA) of the sintered magnet is mainly affected by the grain-boundary structure defect (GBSD). The nanocrystalline magnet is composed of the directly contacted magnetic grains of a few tens of nanometers [3], and its GBA is principally influenced by the intergrain exchange coupling interaction (IECI). However, for the HDDR magnet, its GBA may be simultaneously influenced by GBSD and IECI [4], owing to the unique grain microstructure. Some investigators considered that the adjacent grains directly contacted with each other in the same HDDR powder particle [5,6,7]. However, Nakayama et al [8] observed experimentally that a thin grain-boundary layer with the thickness of $1 \mathrm{~nm}$ exists between adjacent HDDR grains. Theoretically, the effect of intergranular phase (IP) on the coercivity is unclear. Thus, this paper tries to theoretically study the effect of intergranular phase on the coercivity in HDDR Nd-Fe-B magnet.

The component, structure and character of intergranular phase sensitively depend on the alloy's composition and processing technique. The intergranular phase is the crystalline phase with Nd2Fe14B-like structure reported by Reference [9]. Reference [10] pointed out that the $\mathrm{Nd}_{6} \mathrm{Fe}_{13} \mathrm{Al}_{1}$ phase was identified as an intergranular phase. Thus, the intergranular phase is still magnetic phase. Here, assuming that the IP existing between adjacent grains is a weak magnetic phase, and using cubicgrain anisotropy model, we study the effect of IP on the coercivity of the HDDR Nd-Fe-B magnet. The results indicate that the coercivity increases with the increasing IP's thickness $d$, but decreases with increasing its anisotropy constant $K_{1}(0)$. Such conclusion could provide a theoretical reference for preparing high coercivity HDDR Nd-Fe-B magnet.

\section{Anisotropy Model}

Reference [4] pointed out that the GBA is simultaneously influenced by the GBSD and IECI in the HDDR magnet, and proposed a structure model of a cubic grain with edge of $D$ (where the GBSD's thickness is $r_{0}$ and the IECI's length is lex). Here, we assume that the IP is a weak magnetic phase, and distributes homogeneously between grains. Because of the very small size of IP, we presume that half of the thickness, $d / 2$, is shorter than both lex/2 and $r_{0}$ (as shown in Figure 1a where $r_{0}>$ lex/2 is supposed). IP weakens the IECI, leading to the IECI's length reduce from lex/2 to (lex-d)/2. Based on different 
ranges influenced by the GBSD and IECI, a grain is divided into three parts in the case of $D / 2>r_{0}>l e x / 2$. For convenience, the center of IP is chosen as the coordinate origin of $r$. For $d / 2<r<l e x / 2$, the GBA is simultaneously affected by the GBSD and IECI. For $l e x / 2<r<r_{0}$, it is influenced by the GBSD alone. While $r>r_{0}$, the GBA isn't influenced by the GBSD or IECI, and is still the common anisotropy constant $K_{1}$ in the inner part of the grain. The grain-boundary anisotropy $K_{1}(r)$ was described by different formulae for $r_{0} \leq l e x / 2$ and $r_{0}>\operatorname{lex} / 2$ in Reference [4]. Here, we assume that $K_{1}(0)$ is a constant in the IP region. Due to the continuous variation of $K_{1}^{\prime}(r)$, its expression can be rewritten as Equations (1) and (2). Figure 1b shows the variation of $K_{1}{ }^{\prime}(r)$ in the case of $D / 2>r_{0}>$ lex/2. It can be seen that $K_{1}(r)$ continuously decreases from $K_{1}$ in the inner part of a grain to $K_{1}(0)$ in the IP region. when $r_{0} \leq l e x / 2$,

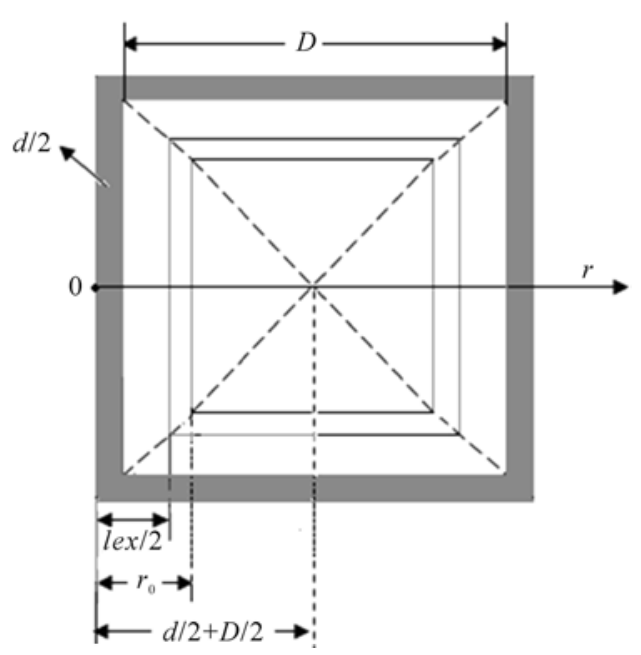

(a)

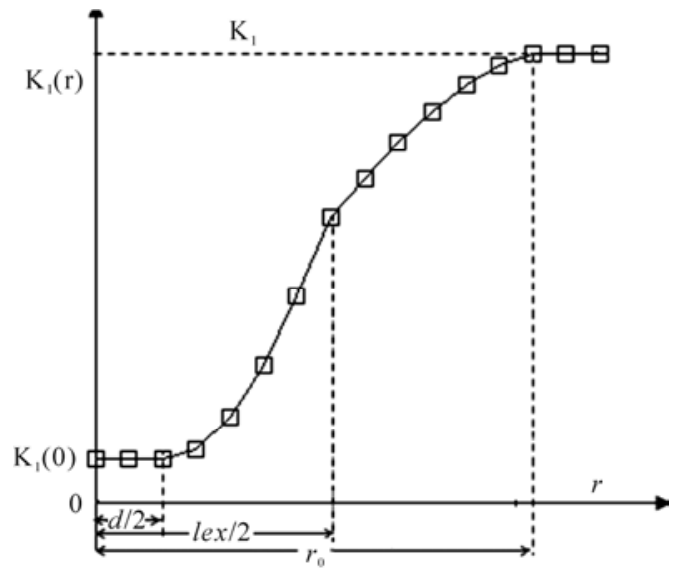

(b)

Figure 1. (a) Sketch of a grain divided into three parts due to different ranges influenced by GBSD and IECI in the case of $D / 2>r_{0}>l e x / 2$; (b) Variation sketch of grainboundary anisotropy

$$
K_{1}^{\prime}(r)= \begin{cases}K_{1}(0), & 0 \leq r \leq \frac{d}{2} \\ K_{1}-\Delta K\left(1-\frac{2\left(r-\frac{d}{2}\right)^{2}}{\left(r_{0}-\frac{d}{2}\right)(l e x-d)}\right)^{\frac{3}{2}}, & \frac{d}{2}<r<r_{0} \\ K_{1}-\Delta K\left(1-\frac{2\left(r-\frac{d}{2}\right)}{(l e x-d)}\right)^{\frac{3}{2}}, & r_{0} \leq r \leq \frac{\text { lex }}{2}\end{cases}
$$

when $r_{0}>\operatorname{lex} / 2$,

$$
K_{1}^{\prime}(r)= \begin{cases}K_{1}(0), & 0 \leq r \leq \frac{d}{2} \\ K_{1}-\Delta K\left(1-\frac{2\left(r-\frac{d}{2}\right)^{2}}{\left(r_{0}-\frac{d}{2}\right)(l e x-d)}\right)^{\frac{3}{2}}, & \frac{d}{2}<r<\frac{l e x}{2} \\ K_{1}-\Delta K\left(1-\frac{\left(r-\frac{d}{2}\right)}{\left(r_{0}-\frac{d}{2}\right)}\right)^{\frac{3}{2}}, & \frac{\text { lex }}{2} \leq r \leq r_{0}\end{cases}
$$

where $r$ is the distance to the IP's center, $\Delta K=K_{1}-K_{1}(0)$, and $K_{1}(0) \leq K_{1}$.

\section{Coercivity of the HDDR Nd-Fe-B Magnet}

The demagnetization process and coercivity mechanism of the HDDR Nd-Fe-B magnet were studied by Reference [4], where the IP didn't exist, and it was concluded that both the demagnetization nucleation and pinning of domain wall displacement between grains might occur at the grain boundary. If IP exists, it might become the pinning center of the domain wall displacement [11]. When the coercivity of magnet is determined by the irreversible domain wall displacement in the IP region, it can be expressed by [12],

$$
H_{c}=\frac{2 K_{1} \pi r_{0}}{3 \sqrt{3} M_{s} \delta_{B}^{\prime}}\left(\frac{A}{A^{\prime}}-\frac{K_{1}^{\prime}}{K_{1}}\right)-N_{e f f} M_{s}
$$

where $A, A^{\prime}$ and $K_{1}, K_{1}{ }^{\prime}$ denote the integral constants and anisotropy constants in the inner and boundary parts of a grain, respectively. $\delta_{\mathrm{B}}$ ' denotes the domain wall thickness. $M_{\mathrm{s}}$ is the saturation magnetization, and $M_{\mathrm{s}}$ in denominator of Equation (3) can be replaced by the saturation polarization $J_{\mathrm{s}}$ in the International System of Units. $N_{\text {eff }}$ is the effective demagnetization factor. 
Reference [12] considered that $A^{\prime}$ is equal to $A$, and $K_{1}{ }^{\prime}$ takes the fixed value less than $K_{1}$. Based on our proposed anisotropy model, $r_{0}$ should be the thickness of anisotropic inhomogeneous district, and is denoted by $\overline{r_{0}}$, and $K_{1}{ }^{\prime}$ varies between 0 and $K_{1}$. For convenience, $K_{1}$ ' in Equation (3) will be replaced by the average anisotropy $\left\langle K_{1}{ }^{\prime}\right\rangle$ in $\bar{r}_{0}$ region. Thus, Equation (3) can be rewritten as,

$$
H_{c}=\frac{2 K_{1} \pi \bar{r}_{0}}{3 \sqrt{3} M_{s} \delta_{B}{ }^{\prime}}\left(\frac{A}{A^{\prime}}-\frac{\left\langle K_{1}^{\prime}\right\rangle}{K_{1}}\right)-N_{\text {eff }} M_{s}
$$

where $<K_{1}{ }^{\prime}>$ can be expressed as follows,

$$
<K_{1}^{\prime}>=\left\{\begin{array}{lc}
\frac{2}{l e x}\left(\int_{0}^{\frac{d}{2}} K_{1}(0) d r+\int_{\frac{d}{2}}^{r_{0}}\left(K_{1}-\Delta K\left(1-\frac{2\left(r-\frac{d}{2}\right)^{2}}{\left(r_{0}-\frac{d}{2}\right)(l e x-d)}\right)^{\frac{3}{2}}\right) d r+\int_{r_{0}}^{\frac{l e x}{2}}\left(K_{1}-\Delta K\left(1-\frac{2\left(r-\frac{d}{2}\right)}{(l e x-d)}\right)^{\frac{3}{2}}\right) d r\right), & r_{0} \leq \frac{l e x}{2} \\
\frac{1}{r_{0}}\left(\int_{0}^{\frac{d}{2}} K_{1}(0) d r+\int_{\frac{d}{2}}^{\frac{l e x}{2}}\left(K_{1}-\Delta K\left(1-\frac{d\left(r-\frac{d}{2}\right)^{2}}{\left(r_{0}-\frac{d}{2}\right)(l e x-d)}\right)^{\frac{3}{2}}\right) d r+\int_{\frac{l e x}{2}}^{r_{0}}\left(K_{1}-\Delta K\left(1-\frac{\left(r-\frac{d}{2}\right)}{\left(r_{0}-\frac{d}{2}\right)}\right)^{\frac{3}{2}}\right) d r\right), & \frac{l e x}{2}<r_{0}
\end{array}\right.
$$

Taking the intrinsic magnetic parameters of $\mathrm{Nd}_{2} \mathrm{Fe}_{14} \mathrm{~B}$ : $K_{1}=4.3 \mathrm{MJ} / \mathrm{m}^{3}, \mathrm{~A}=7.7 \times 10^{-12} \mathrm{~J} / \mathrm{m}, M_{\mathrm{s}}=1280 \mathrm{kA} / \mathrm{m}[13]$, $J_{\mathrm{s}}=1.61 \mathrm{~T}$ [14], lex $=4.2 \mathrm{~nm}[15], \delta_{\mathrm{B}}=4.2 \mathrm{~nm}, N_{\text {eff }}=0.6$ [16], into Equations (4) and (5), we can calculate the coercivity of magnet for different values of $r_{0}, d$ and $K_{1}(0)$.

\section{Results and Discussion}

Figure 2 shows the variations of anisotropy $K_{1}^{\prime}(r)$ for given values of, $r_{0} d$ and $K_{1}(0)$. For different values of $r_{0}$, $d$ and $K_{1}(0), K_{1}^{\prime}(r)$ decreases with decreasing $r$. This is due to that the closer to the grain surface, the smaller the anisotropy is [4]. It can be also seen that, for the fixed $r_{0}$ and $K_{1}(0)$ shown by the star and circle lines, the variation velocities of $K_{1}{ }^{\prime}(r)$ increases with increasing $d$. This is attributed to the decreasing variation range from $d / 2$ to $r_{0}$ with increasing $d$ for the fixed value of $\left(K_{1}-K_{1}(0)\right)$. But for the fixed $r_{0}$ and $d$, shown by the upper triangle and lower triangle lines, the variation velocities of $K_{1}{ }^{\prime}(r)$ decreases with increasing $K_{1}(0)$, which is owing to that, the variation value $\left(K_{1}-K_{1}(0)\right)$ decreases with increasing $K_{1}(0)$ for the fixed variation range from $d / 2$ to $r_{0}$. While for the fixed $d$ and $K_{1}(0)$ shown by the circle and lower triangle lines, the variation speeds of $K_{1}^{\prime}(r)$ decreases with increasing $r_{0}$, attributing to the increasing variation range from $d / 2$ to $r_{0}$ as increasing $r_{0}$ for the fixed value of $\left(K_{1}-\right.$ $\left.K_{1}(0)\right)$.

Figure 3 shows the dependence of average anisotropy, $\left\langle K_{1}^{\prime}>\right.$, on $d$ for different values of $r_{0}$ and $K_{1}(0)$. For different $r_{0}$ and $K_{1}(0),\left\langle K_{1}{ }^{\prime}>\right.$ all decreases with increasing $d$, which is attributed to the variation speeds of $K_{1}^{\prime}(r)$ increases with increasing $d$ (as shown in Figure 2). So, $\left\langle K_{1}{ }^{\prime}\right\rangle$ computed by Equation (5) decreases. But for the fixed $r_{0}$ and $d$ shown by the upper triangle and circle lines, $\left\langle K_{1}{ }^{\prime}\right\rangle$ increases with increasing $K_{1}(0)$, which is owing to the variation velocities of $K_{1}{ }^{\prime}(r)$ decrease with increasing $K_{1}(0)$ (as shown in Figure 2). Thereby, $\left\langle K_{1}{ }^{\prime}\right\rangle$ calculated by Equation (5) increases. It can be also seen that, for the fixed $d$ and $K_{1}(0)$ shown by the upper triangle and square lines, $\left\langle K_{1}{ }^{\prime}>\right.$ increases with increasing $r_{0}$,

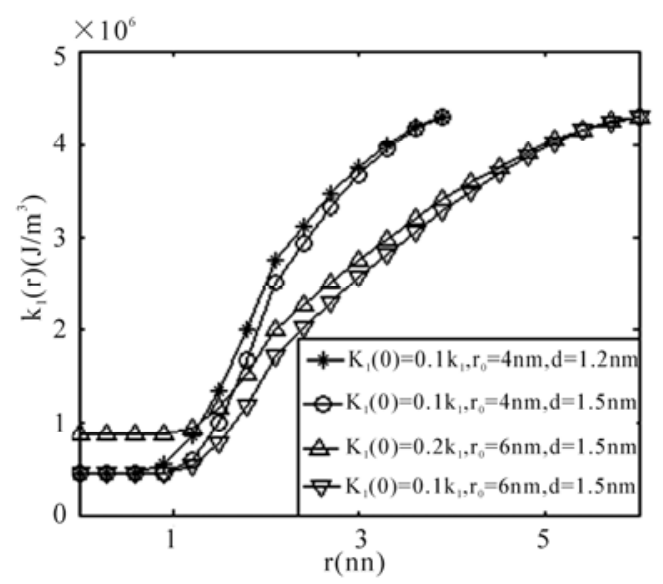

Figure 2. Variations of grain-boundary anisotropy, $K_{1}{ }^{\prime}(r)$, with $r$ for different values of $r_{0}, d$ and $K_{1}(0)$

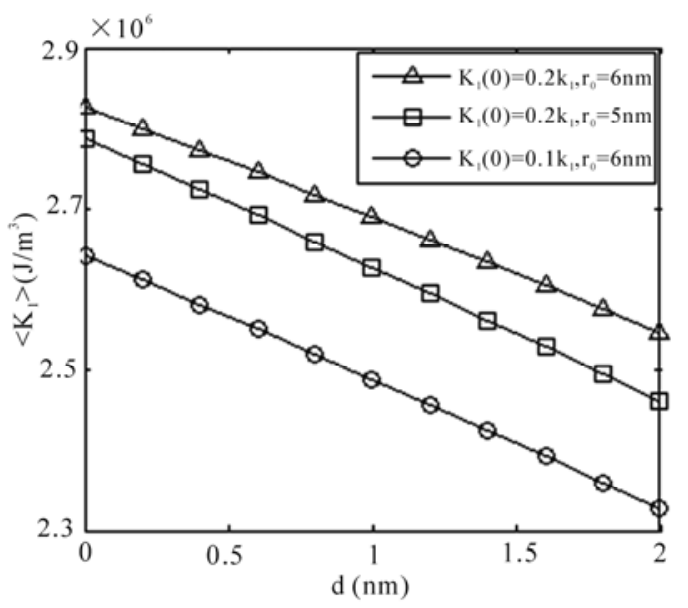

Figure 3. Dependences of average anisotropy, $\left\langle K_{1}{ }^{\prime}\right\rangle$, on $d$ for different values of $r_{0}$ and $K_{1}(0)$ 


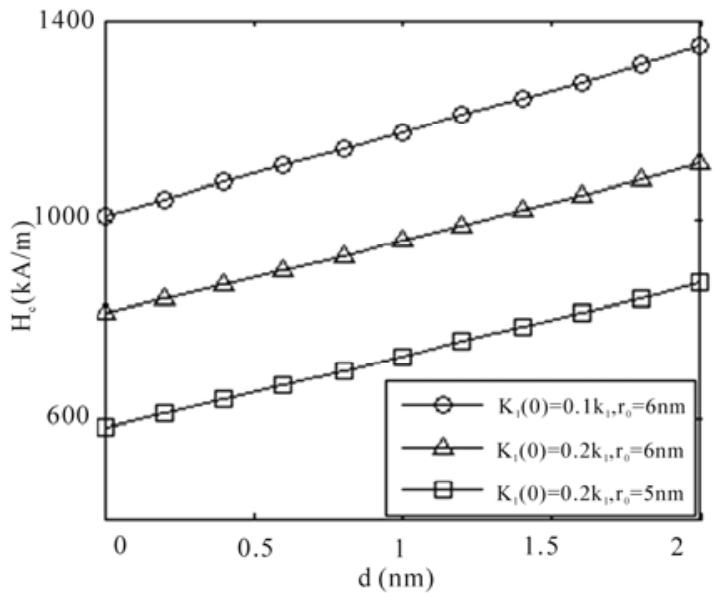

Figure 4. Dependence of Coercivity, $H_{c}$ on $d$ for different values of $r_{0}$ and $K_{1}(0)$

ascribing to the variation speeds of $K_{1}{ }^{\prime}(r)$ decrease with increasing $r_{0}$ (as shown in Figure 2).Thus, $\left\langle K_{1}{ }^{\prime}\right\rangle$ computed by Equation (5) increases.

Figure 4 shows the dependence of coercivity, $H_{c}$, on $d$ for different values of $r_{0}$ and $K_{1}(0)$. For different values of $r_{0}$ and $K_{1}(0), H_{\mathrm{c}}$ increases with increasing $d$. On the one hand, this is owing to that the enhancement of $d$ results in the reduction of $\left\langle K_{1}{ }^{\prime}\right\rangle$ (as shown in Figure 3), and then $H_{c}$ calculated by Equation (4) increases. On another hand, the domain wall energy is the lowest in the IP region, where the domain walls located are the most stable. And the domain walls are pinned more strongly in the IP region with increasing IP's thickness $d$. Thus, a largely external field is needed if the domain walls tend to deviate from the IP region, and then $H_{\mathrm{c}}$ also increases. But for the fixed $r_{0}$ and $d$ shown by the circle and upper triangle lines, $H_{\mathrm{c}}$ decreases with increasing $K_{1}(0)$. On the one hand, for the fixed $r_{0}$ and $d,\left\langle K_{1}{ }^{\prime}\right\rangle$ increases with increasing $K_{1}(0)$ (as shown in Figure 3). Thus $H_{\mathrm{c}}$ computed by Equation (4) decreases. On another hand, with increasing $K_{1}(0)$, the pinning force hindering the moving of domain wall becomes smaller, thus the domain wall deviates from the IP region more easily. So, the coercivity decreases. It can be also seen that, for the fixed $K_{1}(0)$ and $d$ shown by the upper triangle and square lines, $H_{\mathrm{c}}$ increases with increasing $r_{0}$, attributing to the variable quantities of $\frac{\bar{r}_{0}}{\delta_{B}}$ is larger than that of $\left(\frac{A_{1}}{A_{1}^{\prime}}-\frac{\left\langle K_{1}^{\prime}\right\rangle}{K_{1}}\right)$ with increasing $r_{0}$. Consequently, $H_{\mathrm{c}}$ computed by Equation (4) increases. When $r_{0}=6 \mathrm{~nm}, d=1 \mathrm{~nm}$ and $K_{1}(0)=$ $0.15 K_{1}$, the calculated coercivity is $1068 \mathrm{kA} / \mathrm{m}$, which is consistent well with the experimental data (IP's thickness of the HDDR Nd-Fe-B magnet is around $1 \mathrm{~nm}$, and its coercivity is $1058 \mathrm{kA} / \mathrm{m}$ ) reported by Nakayama et al [8].

In summary, the weak magnetic intergranular phase (IP) existing between adjacent grains weakens the IECI.
The increase of both the IP's thickness $d$ and GBSD's thickness $r_{0}$ or the decrease of the IP's anisotropy constant $K_{1}(0)$ all enhance the coercivity of magnet. Yet, if $d$ and $r_{0}$ are too larger and $K_{1}(0)$ is too smaller, the magnetization and remanence would badly fall, then it is impossible to obtain high-energy product. In order to get high-energy product, it needs not only to enhance coercivity, but also to keep a sufficiently high remanence. Therefore, it is necessary to ensure that the IP's thickness is around $1 \mathrm{~nm}$, the GBSD's thickness is around $6 \mathrm{~nm}$, and $K_{1}(0)$ varies between $0.1 K_{1}$ and $0.2 K_{1}$, by reasonably adjusting the alloy's composition and technical process. So, this paper possesses a high preference value for experiment preparing high coercivity HDDR Nd-Fe-B magnet with considerable magnetization and remanence.

\section{Conclusions}

Effects of the IP's thickness $d$, its anisotropy constant $K_{1}(0)$, and the GBSD's thickness $r_{0}$ on the coercivity in the HDDR Nd-Fe-B magnet are investigated. The results indicate that $H_{\mathrm{c}}$ increases with the increasing $d$ and $r_{0}$, but decreases with the increasing $K_{1}(0)$. And while $r_{0}=6$ $\mathrm{nm}, d=1 \mathrm{~nm}$ and $K_{1}(0)=0.15 K_{1}$, the calculated coercivity is consistent well with experimental data.

\section{Acknowledgements}

The work is supported by the National Natural Science Foundation of China (50671055) and (50801043).

\section{REFERENCES}

[1] H. Nakamura, R. Suefuji, S. Sugimoto, M. Okada, and M. Homma, "Effects of HDDR treatment conditions on magnetic properties of Nd-Fe-B anisotropic powders," Journal Applied Physics, Vol. 76, No. 10, pp. 6828-6830, November 1993.

[2] M. Sagawa, S. Fujimura, H. Yamamoto, Y. Matsuura, and K. Hiraga, "Permanent magnet materials based on the rare earth-iron-boron tetragonal compounds,” IEEE Transactions on Communications, Vol. 20, No. 1, pp. 1584 1589, January 1984.

[3] R. K. Mishra, "Microstructure of melt-spun Nd-Fe-B magnequench magnetis,” Journal of Magnetism and Magnetic Materials, Vol. 54, No. 1, pp. 450-456, February 1986.

[4] M. Liu, Y. Sun, G. B. Han, Wu. Y, and R. W. Gao, "Dependence of anisotropy and coercivity on microstructure in HDDR Nd-Fe-B magnet," Journal of Alloys Compounds, Vol. 478, pp. 303-307, April 2009.

[5] T. Takeshita and R. Nakayama, "Magnetic properties and microstructures of the NdFeB magnet powder produced by hydrogen treatment," Proceedings of the 10th International Workshop on Rare-Earth Magnets and Their Applications, Kyoto, Japan, pp. 551, 1989.

[6] T. Takeshita, K. Morimoto, “Anisotropic Nd-Fe-B bonded magnets made from HDDR powders,” Journal 
Applied Physics, Vol. 79, No. 8, pp. 5040-5044, April 1996.

[7] R. W. Gao, J. C. Zhang, and D. H. Zhang, "Dependence of the magnetic properties on the alignment magnetic field for Nd-Fe-B bonded magnets made from anisotropic HDDR powders,” Journal of Magnetism and Magnetic Materials, Vol. 191, No. 1, pp. 97-100, January 1999.

[8] R. Nakayama, T. Takeshita, and M. Itakura, "Microstructures and crystallographic orientation of crystalline grains in anisotropic Nd-Fe-Co-B-(Ga or $\mathrm{Zr}$ ) magnet powders produced by the hydrogenation-decomposition-desorption-recombination process," Journal Applied Physics, vol. 76, No. 1, pp. 412-417, July 1994.

[9] Q. G. Ji, B. X. Gu, J. R. Zhang, Z. J. Tian, Y. W. Du, "Effects of aging at room temperature on as-spun NdFe B/a-Fe nanocomposite magnets,” Journal of Magnetism and Magnetic Materials, Vol. 288, No. 2, pp. 84-91, January 2005.

[10] J. Fidler and T. Schrefl, "Overview of Nd-Fe-B magnets and coercivity,” Journal Applied Physics, Vol. 79, No. 2, pp. 5029-5034, September 1996.

[11] M. Yue, X. B. Liu, Y. F. Xiao, and J. X. Zhang, “Magnetization reversal mechanism of anisotropic HDDR
$\mathrm{Nd}_{2} \mathrm{Fe}_{14} \mathrm{~B}$-based magnet powder,” Journal of Magnetism and Magnetic Materials, Vol. 269, No. 2, pp. 227-230, February 2004.

[12] H. Kronmller, K. D. Durst, and M. Sagawa, “Analysis of the magnetic hardening mechanism in RE-Fe-B permanent magnets,” Journal of Magnetism and Magnetic Materials, Vol. 74, No.3, pp. 291-302, February 1988.

[13] H. Kronmller, M. Fischer, and R. Seeger, "Micromagnetism and microstructure of hard magnetic materials," Journal Physics D, Vol. 29, No. 2, pp. 2274-2283, January 1996.

[14] M. Sagawa and S. Fujimura, "New material for permanent magnets on a base of $\mathrm{Nd}$ and Fe," Journal Applied Physics, Vol. 55, No. 6, pp. 2083-2087, March 1984.

[15] G. B. Han and R. W. Gao, "Effect of exchange-coupling interaction on the effective anisotropy in nanocrystalline $\mathrm{Nd}_{2} \mathrm{Fe}_{14} \mathrm{~B}$ material," Journal of Magnetism and Magnetic Materials, Vol. 281, No. 1, pp. 6-10, October 2004.

[16] M. GrÄonefeld and H. Kronmller, "Calculation of strayfields near grain edges in permanent magnet material," Journal of Magnetism and Magnetic Materials, Vol. 80, No. 2, pp. 223-228, August 1989. 